Apidologie, 1981, 12 (2), 185-207.

\title{
RASTERELEKTRONENMIKROSKOPISCHE UNTERSUCHUNGEN ÜBER DIE ONTOGENIE VON NOSEMA APIS ZANDER
}

\author{
Wolfgang STECHE und Theo HELD \\ Landesanstalt für Bienenkunde, Universität Hohenheim \\ August von Hartmann Str. 13, D-7000 Stuttgart 70 (B.R.D.)
}

\begin{abstract}
SUMMARY
SCANNING ELECTRON MICROSCOPE STUDY OF THE ONTOGENESIS OF NOSEMA APIS ZANDER

To clarify the question of the developmental phases of Nosema apis Zander in which divisions of the parasitic stages occur, the pathogen in its various periods of development was examined in the intestinal epithelium of infected bees with the electron scanning microscope. Parallel to this, different stages were examined in smear preparations. It was shown that divisions only occur in a very early stage not detectable optically.

These dividing stages are partially liberated in the intestinal lumen and migrate into the epithelial cells. This explains the simultaneous infection of several adjacent cells by one spore.

The primary stages pass through the polar thread and must consist almost exclusively of D.N.A.

On the basis of the conditions described, the designation Nosema apis Zander is correct according to the systematic classification of the microsporidia by J. WEISER.
\end{abstract}

\section{ZUSAMMENFASSUNG}

Um die Frage zu klären, in welchen Entwicklungsphasen von Nosema apis Zander Teilungen der Parasitenstadien erfolgen, wurde der Erreger in seinen verschiedenen Entwicklungsabschnitten im Darmepithel infizierter Bienen rasterelektronenmikroskopisch untersucht. Parallel dazu erfolgte die Prüfung der verschiedenen Stadien in Ausstrichpräparaten. Es zeigte sich, dass Teilungen nur in einem sehr frühen, lichtoptisch nicht fassbaren Stadium erfolgen. 
Diese Teilungsstadien werden teilweise bereits im Darmlumen freigesetzt und wandern in die Epithelzellen ein. Dadurch lässt sich die gleichzeitige Infektion mehrerer benachbarter Zellen durch eine Spore erklären.

Die Primärstadien durchwandern den Polfaden und dürften fast ausschliesslich aus D.N.S. bestehen.

Aufgrund der geschilderten Verhältnisse ist nach der systematischen Gliederung der Mikrosporidia von J. WeISER die Bezeichnung Nosema apis Zander zutreffend.

\section{EINLEITUNG}

In dem Bemühen, die Ontogenie von Nosema apis zu klären, standen dem Sachbearbeiter bisher zwei Analyseverfahren zur Verfügung, das histologische Präparat und der Ausstrich.

Beide Verfahren führten zu der Erkenntnis, dass der Parasit im Laufe seiner Entwicklung verschiedene Stadien durchläuft, wobei sich in diesen Stadien unterschiedliche Kernprozesse abspielen. Die so wichtige Frage, wann und wo Teilungs- und damit Vermehrungsphasen im Entwicklungsablauf eingeschaltet sind, wurde dabei bisher unterschiedlich und somit unbefriedigend beantwortet (JACOBS, 1976).

Der gewählte Terminus “ Meront " lässt bereits die Annahme erkennen, dass sich vor der Bildung der Sporonten und Sporoblasten (FAnTham u. Porter, 1912) die Stadien in einer, wie man annahm, lichtoptisch fassbaren Grösse teilen, und zwar so lange, bis das Plasma der Zelle verbraucht und damit die Zelle vollständig mit Stadien ausgefüllt ist.

Weitere Teilungsprozesse wurden in den dem Merontenstadium vorausgehenden Planonten- Schizontenstadien gesehen.

Wesentlich für die systematische Einordnung des Parasiten innerhalb der Gruppe der Mikrosporidia war und ist zudem die Frage, ob es vor der Sporenbildung nochmals $\mathrm{zu}$ einer Teilung der Sporonten in Sporoblasten kommt oder nicht.

Um eine Klärung dieser meist als Hypothesen geässerten widersprüchlichen Ansichten über die Teilung der Entwicklungsstadien herbeiführen zu können, bemühten wir uns, mit Hilfe des Rasterelektronenmikroskopes die Frage der Zell- oder Stadienteilungen im Entwicklungsverlauf des Parasiten exakt beantworten zu können.

Dieses Analyseverfahren bietet die Möglichkeit, nach einer harten Fixierung die jeweilige Lage der Stadien im umgebenden Medium und ihre äussere Form genau beschreiben zu können. Der Nachteil des Verfahrens besteht allerdings darin, dass irgendwelche Aussagen über die Entwicklungsvorgänge innerhalb der Stadien, speziell der Kernprozesse nicht möglich sind. 


\section{METHODIK}

Der Versuch, die Entwicklungsstadien des Nosemaerregers rasterelektronenmikroskopisch zu analysieren, wurde 1977 in Angriff genommen. Im Rahmen dieser Arbeit wurden Tausende von infizierten Zellen untersucht.

\section{Ziehen der Därme}

Den mit $\mathrm{CO}_{2}$ betäubten Bienen wird der Thorax eingedrückt und mit Hilfe einer Pinzette der Mitteldarm gezogen.

\subsection{Polfadenpräparate}

Die gereinigten Sporen werden in Insektenringerlösung $\left(7,5 \mathrm{~g} \mathrm{NaCl}, 0,35 \mathrm{~g} \mathrm{KCl}, 0,21 \mathrm{~g} \mathrm{CaCl}_{2}\right.$ in $1000 \mathrm{ml} \mathrm{H} \mathrm{H}_{2} \mathrm{O}$ ) suspendiert und bei $35^{\circ} \mathrm{C} 15-30$ Minuten im Thermostat gehalten. Anschliessend kommen die Deckgläschen mit den inzwischen sedimentierten, darauf anhaftenden Stadien in einen schwach fliessenden Wasserstrom und werden dann wie folgt weiterbehandelt.

\section{Fixierung}

Die Därme bzw. Ausstriche kommen sofort in eine phosphatgepufferte $4 \%$ Glutaraldehyd-Lösung und werden 2 Stunden bei $4^{\circ} \mathrm{C}$ im Kühlschrank fixiert.

Ansatz der Pufferstammlösung :

Natrium und Kaliumphosphat sind $0,066 \mathrm{~m}$.

$1,1 乞 \mathrm{~g} \mathrm{Na}_{2} \mathrm{HPO}_{4} \times 2 \mathrm{H}_{2} \mathrm{O} / 100 \mathrm{ml} \mathrm{H} \mathrm{H}_{2} \mathrm{O}$.

$0,91 \mathrm{~g} \mathrm{KH}_{2} \mathrm{PO}_{4} / 100 \mathrm{ml} \mathrm{H}_{2} \mathrm{O}$.

Ansatz der Pufferlösung : Mischung von 71,5 $\mathrm{ml} \mathrm{Na}_{2} \mathrm{HPO}_{4}$ und 28,5 $\mathrm{ml} \mathrm{KH}_{2} \mathrm{PO}_{1}$. Die $4 \%$ ige Glutaraldehydlösung ist täglich neu anzusetzen.

Anschliessend wird zweimal mit Phosphatpuffer und zweimal mit aqua dest. gespült. Minuten).

Nun werden die Präparate durch die steigende Acetonreihe geführt (10\% Schritte, pro Schritt 15

\section{Critical Point Trocknung}

Die in reinem Aceton liegenden Därme bzw. Ausstriche, werden im Critical Point Dryer 11120 von Balzers Union getrocknet. Hierbei wird das Aceton durch $\mathrm{CO}_{2}$ ersetzt und die Därme bei $85 \mathrm{Atm}$. Druck und $40^{\circ} \mathrm{C}$ unter Umgehung des kritischen Punktes auf schonende Art und Weise getrocknet.

\section{Präparieren und Brechen der Därme}

Unter dem Binocular werden die Malpighischen Gefässe entfernt und die Därme je nach Bedarf quer oder längs gebrochen.

\section{Aufkleben auf Aluminium-Blöcke}

Die Aluminium-Blöcke werden mit einer dünnen Leitsilberschicht bestrichen und die Darmstücke nach kurzer Wartezeit in die plastische Masse eingebracht und einige Stunden getrocknet.

\section{Besputtern}

Mit Hilfe des Sputter Coaters S 150 von Edwards wird nun die Präparat-Oberfläche mit einer leitenden Goldschicht versehen. Bei $760 \times 10^{-1}$ Torr und $11 \mathrm{mV}$ hat sich bei Därmen eine Sputterzeit von 4 Minuten, bei Ausstrichen auf Glasunterlagen von 6 Minuten als optimal erwiesen.

\section{Rasterelektronische Untersuchung}

Die Präparate wurden mit den Jeol J.S.M. 35 Rasterelektronenmikroskop des Instituts für Physik in Hohenheim analysiert.

Den Direktoren des physikalischen Institutes der Universität Hohenheim, den Herren Professoren Dr. Rentschler und Dr. SeIler, sowie Frau Dr. I. Rentschler danken wir für das Einverständnis, diese Untersuchungen in ihrem Institut durchführen zu können. Fräulein I. PLöCHL und Frau M. HEPP danken wir für die sachkundige Unterstützung bei der Arbeit an dem Gerät. 


\section{ERGEBNISSE}

Im nachfolgenden Abschnitt sind die als Beleg dienenden ausgewählten Abbildungen zusammengefasst.

Die Abbildungen 1- 7 zeigen Sektoren von peritrophischen Hüllen, auf denen Polfäden, bzw. die daraus austretenden Primärstadien liegen. In Abbidung 2 und 3 ist zu erkennen, wie ein Polfaden jeweils durch das feine, unregelmässig strukturierte Netz dieser Hüllen (Streuungstextur nach PETERS, 1968), durchgewandert ist, bzw. die in den Hüllen unregelmässig angeordneten offenen Stellen (Abb. 1) zum Durchwandern genutzt hat.

Die Abbildungen 4-7 zeigen, wie sich im vorderen Bereich des Polfadens kugelförmige Stadien abzuschnüren beginnen. Offensichtlich löst sich danach die Mantelsubstanz des Filamentes durch die im Darmlumen enthaltenen Fermente auf, so dass die Einzelstadien frei auf der peritrophischen Hülle liegen bleiben.

Die Tatsache, dass sich Polfäden nach einer gewissen Zeit auflösen, lässt sich auch im Lichtmikroskop beobachten. Bei Ausstrichpräparaten im Phasenkontrastoder Dunkelfeldverfahren betrachtet, sind zunächst gut ansprechbare Polfäden nach etwa einer Stunde nicht mehr sichtbar.

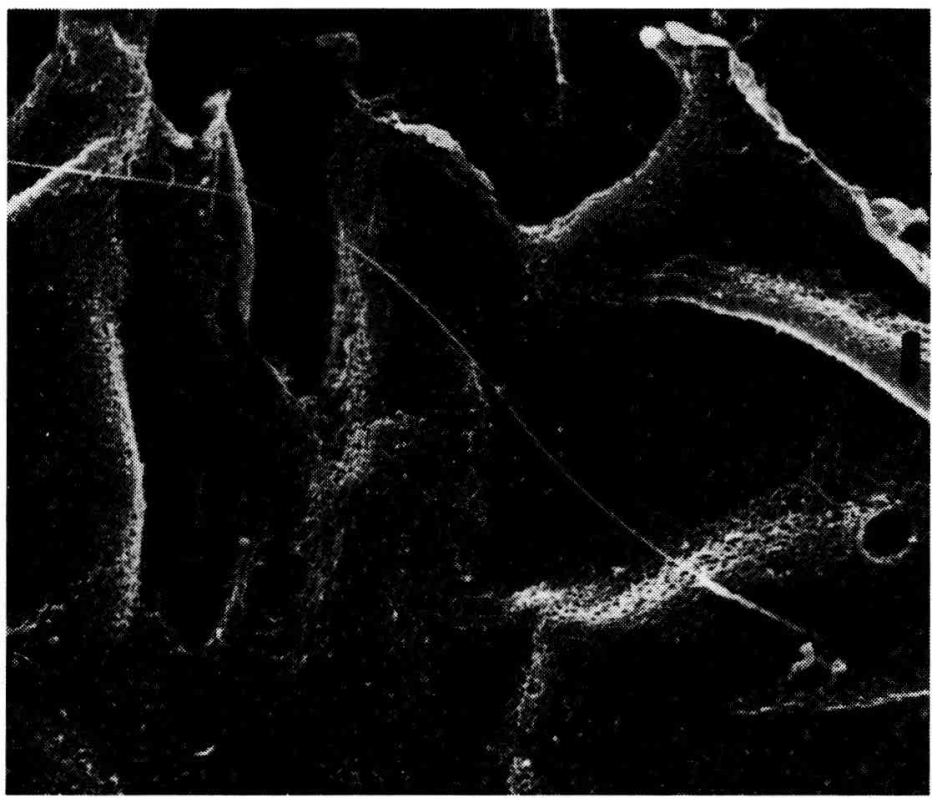

Авв. 1. - Polfaden auf peritrophischer Membran. $1 \mu \mathrm{m}$

FIG. 1. - Filament polaire sur la membrane péritrophique. $1 \mu \mathrm{m}$ 


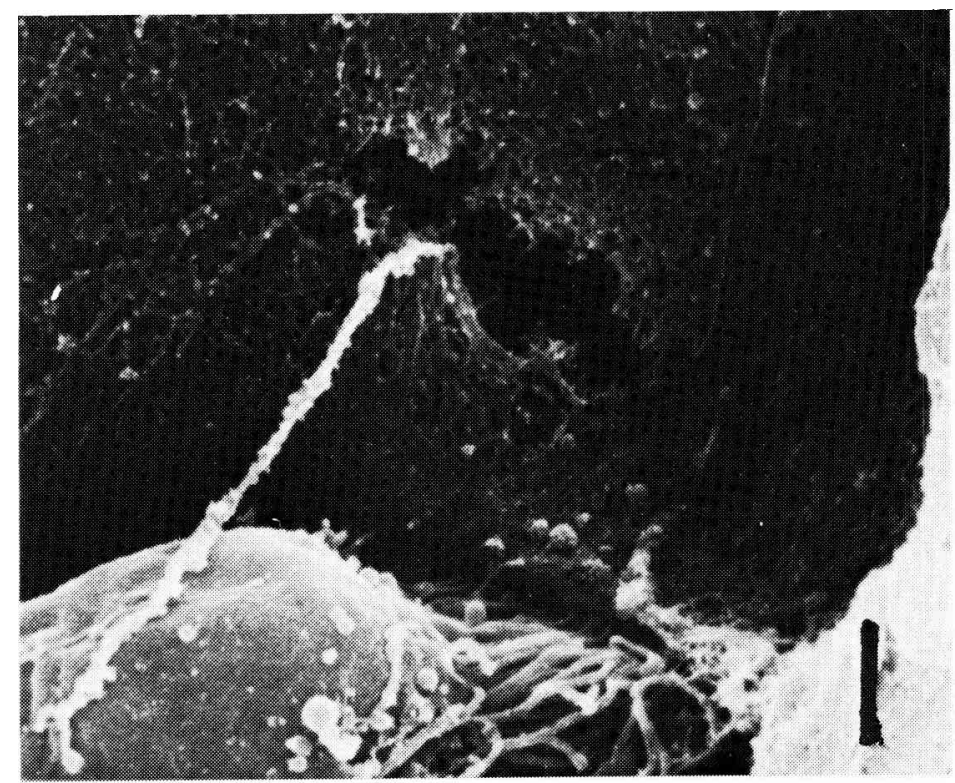

Авв. 2. - Polfaden, der durch einen Riss einer peritrophischen Membran hindurch gewandert ist. $\longrightarrow 1, \mu \mathrm{m}$

FIG. 2. - Filament polaire qui traverse une rupture d'une membrane péritrophique.

$\longrightarrow 1 \mu \mathrm{m}$

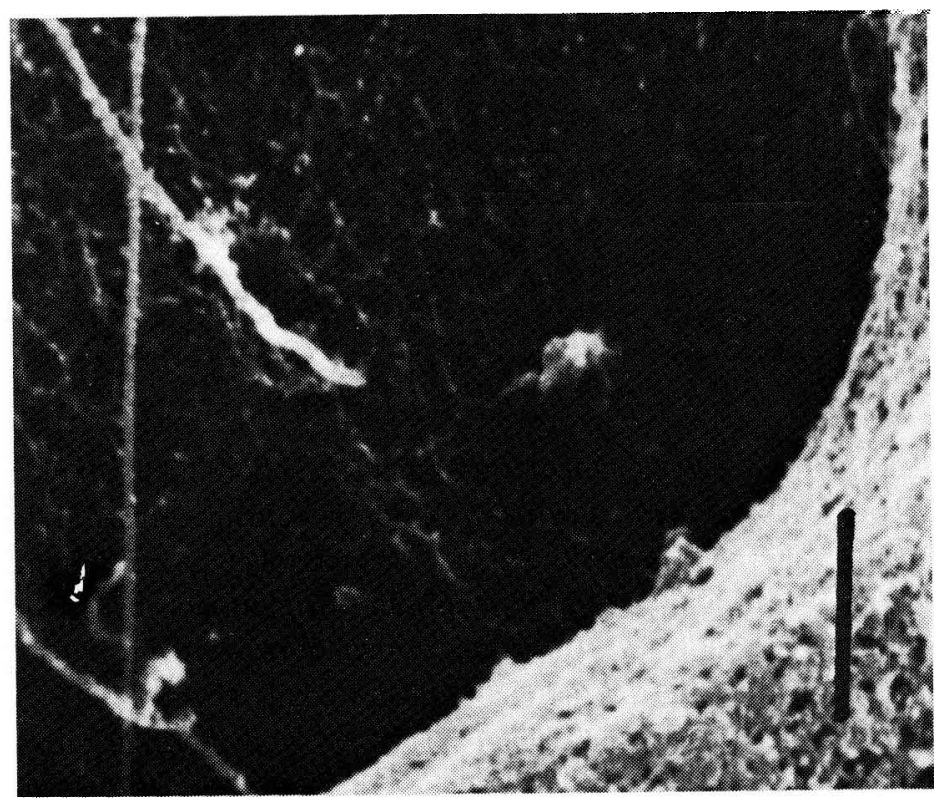

Авв. 3. - Siehe Abb. 2.

$1 \mu \mathrm{m}$

FIG. 3. - Voir Fig. 2. 


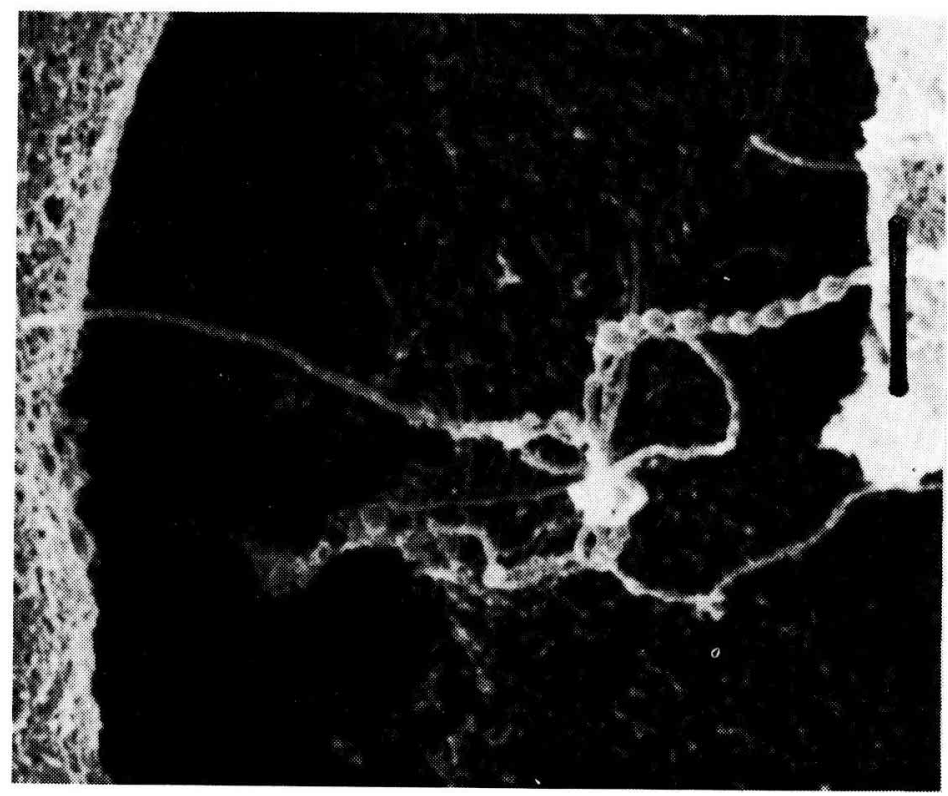

Aв8. 4. - Polfadenspitze, aus der sich Primärstadien entwickeln. $1 \mu \mathrm{m}$

FIG. 4. - Extrémité d'un filament polaire, à partir de laquelle se développent les stades primaires.

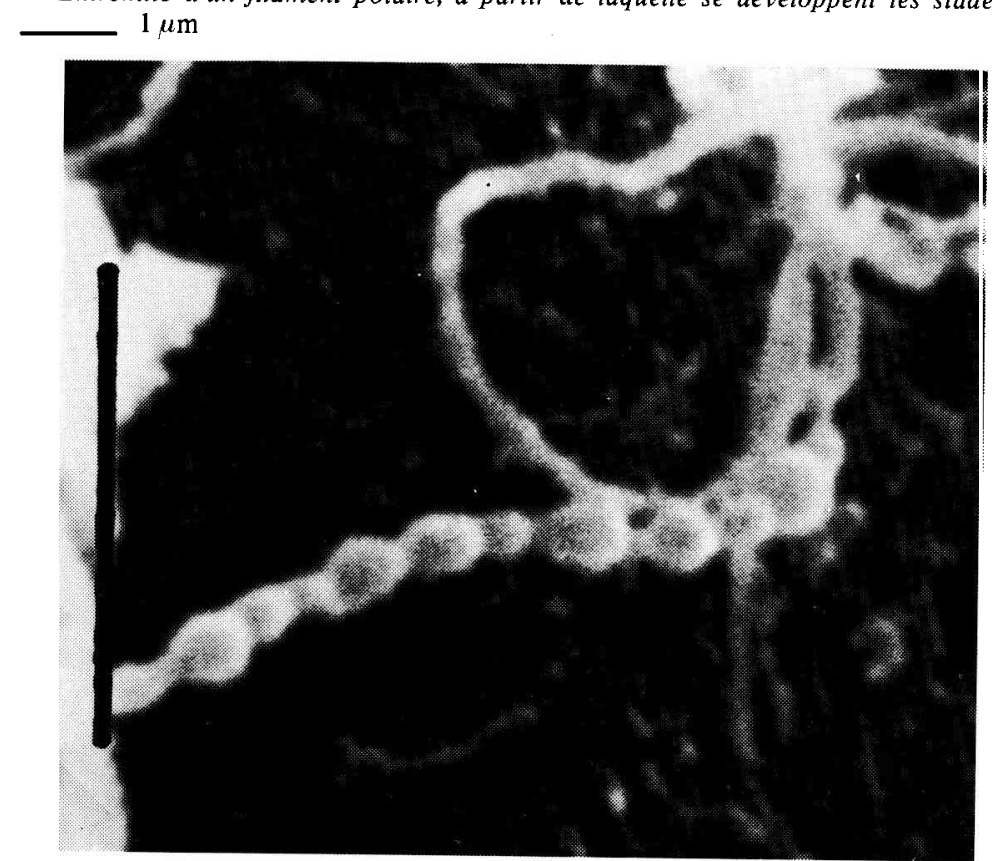

ABB. 5. - Ausschnittvergrösserung aus Abb. 4.

$1 \mu \mathrm{m}$

FIG. 5. - Grossissement d'une portion de la Fig. 4. $1 \mu \mathrm{m}$ 


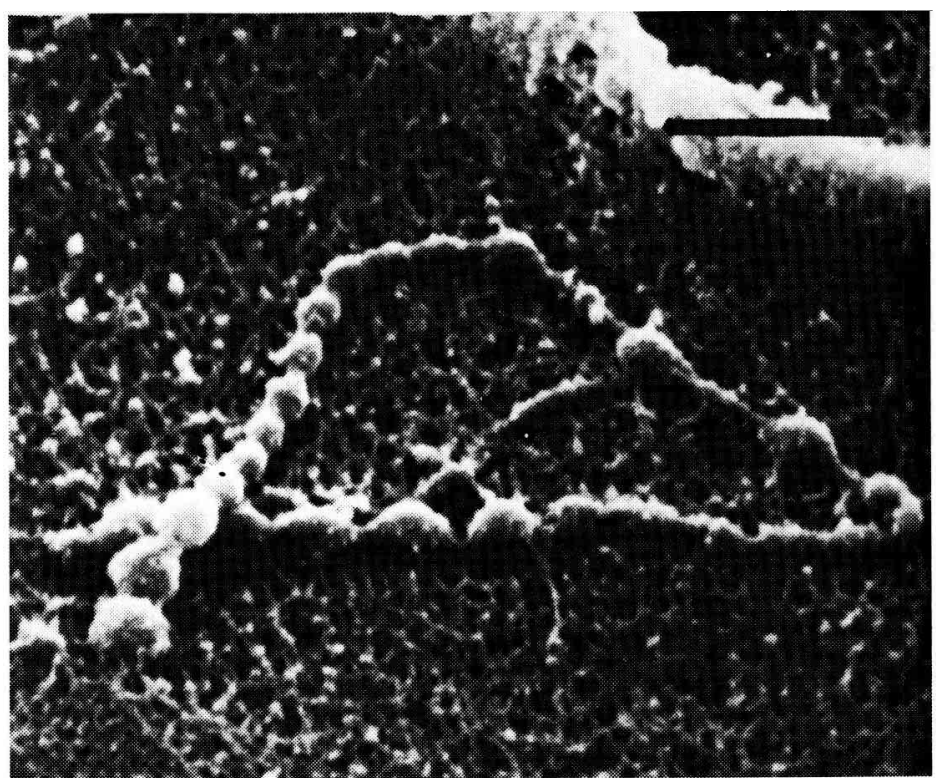

Авв, 6. - Siehe Abb. 4.

$1 \mu \mathrm{m}$

FIG. 6. - Voir Fig. 4.

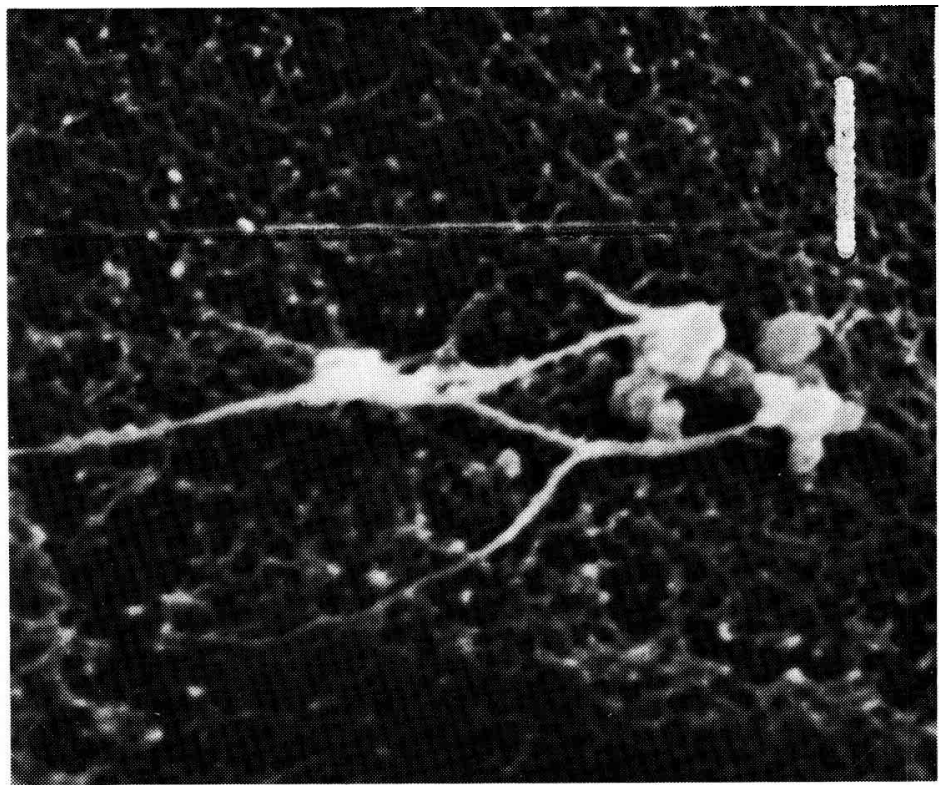

Aвв. 7. - Primäre Teilungsstadien, auf einer peritrophischen Membran liegend. $1 \mu \mathrm{m}$

FIG. 7. - Stades primaires de développement, situés sur une membrane péritrophique. $1 \mu \mathrm{m}$ 
Wahrscheinlich sind es die gleichen Stadien, die STECHE (1962) als "Spiralstadien " beschrieben hat, bei denen er ebenfalls Teilungsprozesse glaubte annehmen zu können. Bei Anfärbung der Ausstriche mit GIEMSA konnte er seinerzeit zeigen, dass diese lichtoptisch nur unter grössten Schwierigkeiten zu fassenden Stadien völlig frei von jeglichem Plasmamantel mehr oder weniger ausschliesslich aus D.N.S. aufgebaut waren.

Um einen genaueren Einblick der Freisetzung dieser Primärstadien aus dem Filament erhalten zu können, wurden wie beschrieben, Ausstrichpräparate von ausgetretenen Polfäden angefertigt. Dabei gelang in einigen wenigen Fällen der Nachweis, dass die Substanz, die im Filament an die Spitze des Polfadens wandert, in einzelne Partien unterteilt ist (siehe Abbildung 8-10). Zudem wurde erkennbar, dass bei Beschädigung des Polfadens an den Bruchstellen Substanz aus dem Schlauch austritt (siehe Abbildung 9 und 10). Darüber hinaus konnte beobachtet werden, dass an der Spitze des Polfadens Substanz austritt und sich sodann in kleinste Partikel differenziert (siehe Abbildung 11 und 12). Diese Beobachtungen erhärten die Annahme, dass die in den präparierten Bienen gefundenen Primärstadien auf diese aus den Filamenten freigesetzte Substanz zurückzuführen sind.

In Verfolgung des weiteren Werdeganges der Primärstadien zeigte es sich, dass sie offensichtlich durch das Flimmerepithel und die Darmzellwand in die Zellen

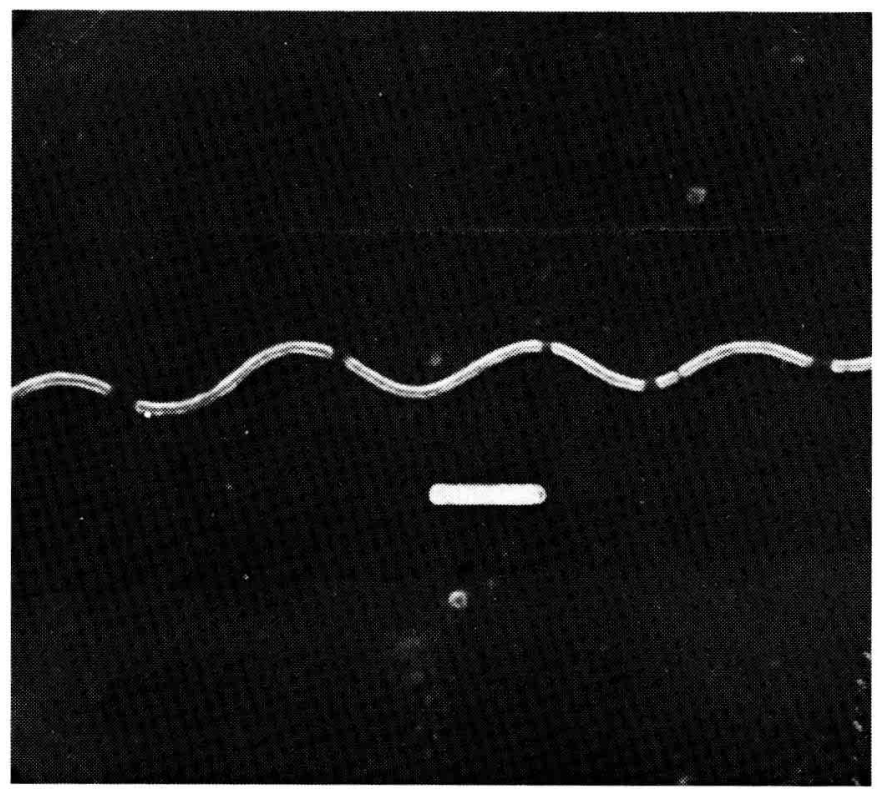

Авв. 8. - Polfadenspitze, in welcher der segmentierte Inhalt zu erkennen ist. $1 \mu \mathrm{m}$

FIG. 8. - Extrémité d'un flament polaire dans laquelle on peut reconnaitre le contenu segmenté. $1 / 1 \mathrm{~m}$ 


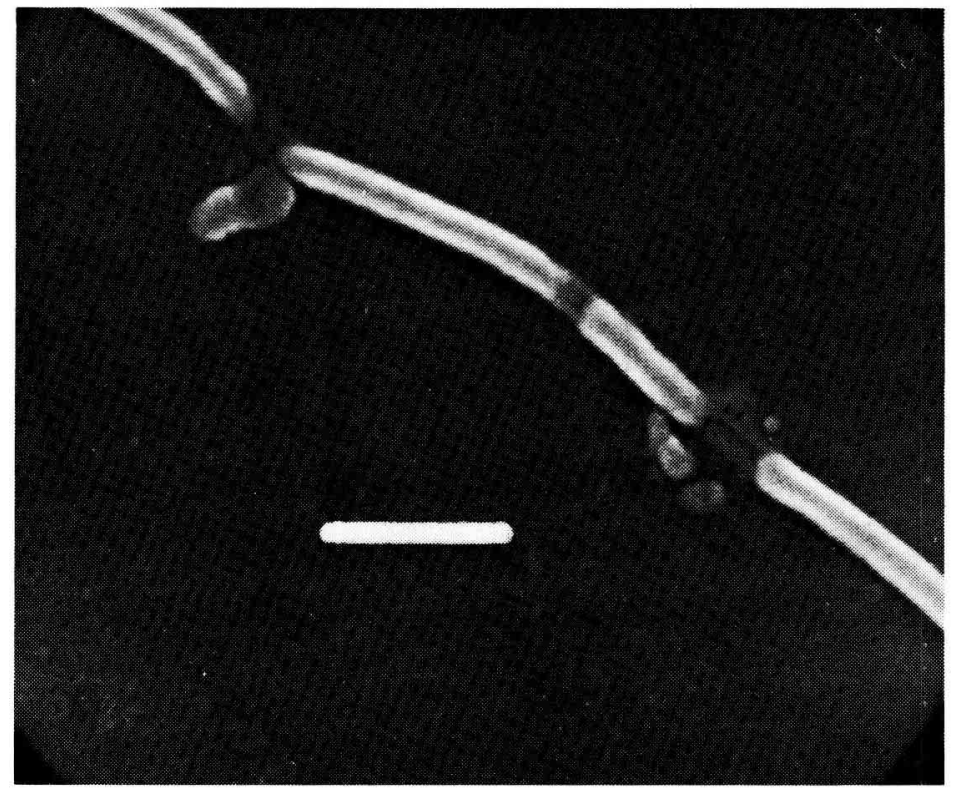

Aвв. 9. - Polfaden, bei welchem an 2 Bruchstellen der Inhalt austritt. $1 \mu \mathrm{m}$

Fig. 9. - Filament polaire présentant 2 cassures desquelles sort le contenu. $1 \mu \mathrm{m}$

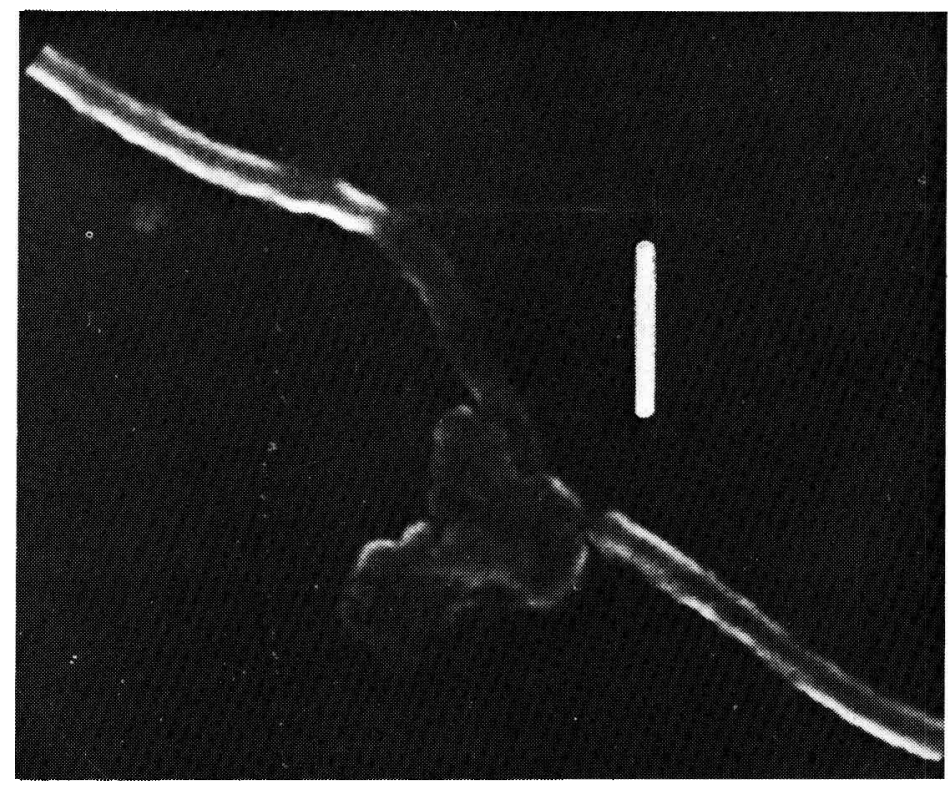

$1 \mu \mathrm{m}$

Авв. 10. - Siehe Abb. 9.

FIG. 10. - Voir Fig. 9. 


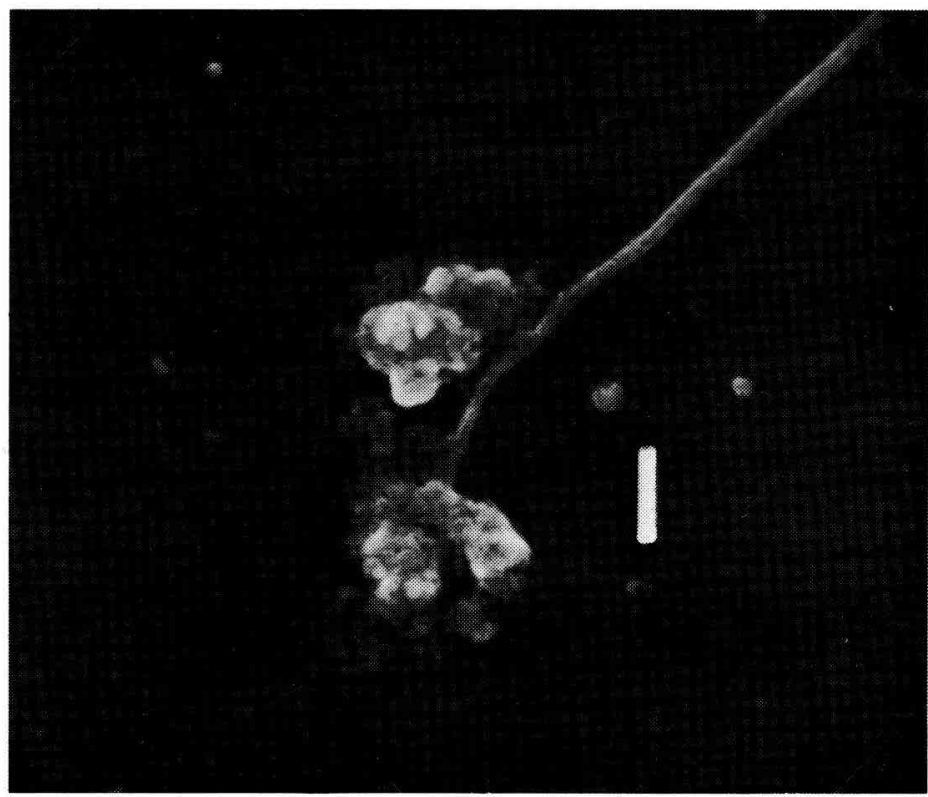

Aвв. 11. - Primärstadien aus der Polfadenspitze austretend. $1 \mu \mathrm{m}$

FIG. 11. - Stades primaires sortant de l'extrémité du filament polaire. $1 \mu \mathrm{m}$

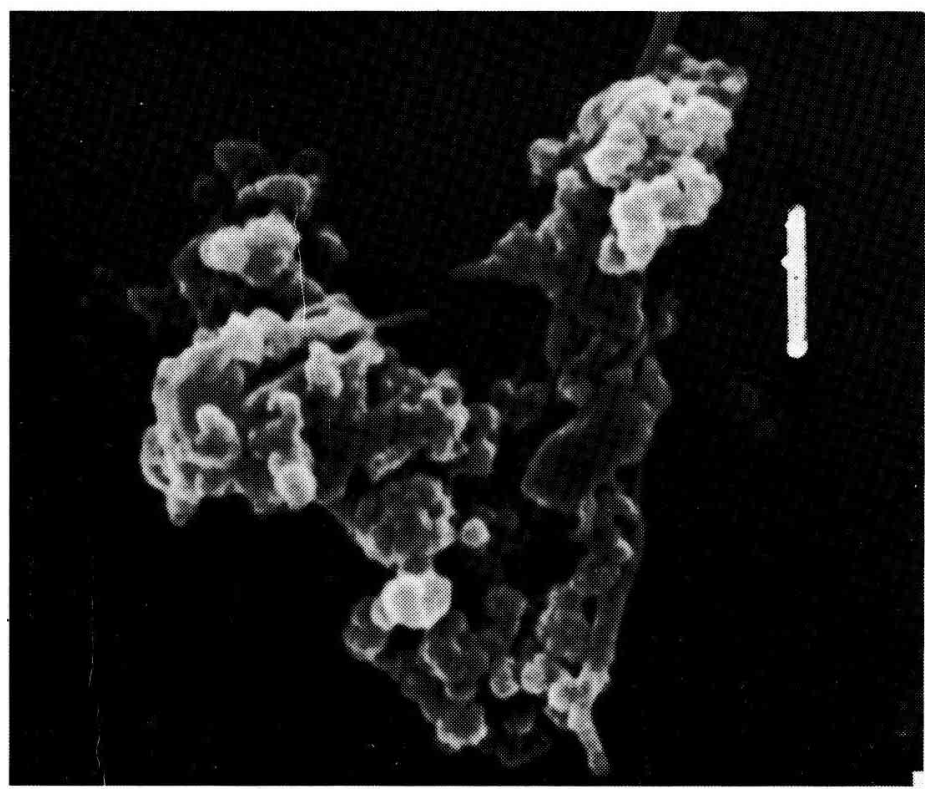

Авв. 12. - Siehe Abb. 11 .

$1 \mu \mathrm{m}$

Fig. 12. - Voir Fig. 11 . 
einzudringen vermögen (siehe Abbildung 13, 14 und 15), um sich dann zu "Kugelstadien " mit zunehmender Grösse zu entwickeln. Dabei dringen sie offensichtlich durchaus auch in bereits infizierte Zellen ein, wie die Abbildungen 16 und 17 beweisen. Ob diese Kugelstadien sich danach nochmals zu teilen vermögen, ist mit Sicherheit kaum zu entscheiden. Typische Teilungsstadien liessen sich nicht ein einziges Mal beobachten. Andererseits fiel auf, dass in infizierten Zellen Kugelstadien unterschiedlicher Grösse zu finden waren (Siehe Abbildung 18).

Der Übergang von den Kugelstadien zu denjenigen Stadien, die eine sporenartige Form annehmen, erfolgt fliessend, wobei man wohl davon ausgehen darf, dass sich hierbei diverse Kernprozesse vollziehen.

Dass diese sporenartigen Stadien unterschiedlich aufgebaut sind, wurde bei der Anfertigung von Ausstrichpräparaten infizierter Zellen erkennbar, die erst nach leichtem Antrocknen fixiert wurden. Es zeigte sich nämlich, dass etliche der rund- oder sporenförmigen Stadien, bei letzteren insbesondere diejenigen, die grösser und vor allem breiter als die adulten Sporen waren, dass diese Stadien bei dem leichten Antrocknen zusammenfielen und im peripheren Bereich einschrumpften (siehe Abbildung 19 bis 21). Demnach haben Stadien dieser Entwicklungsphase nur eine hauchdünne, wenig widerstandsfähige umgebende Membran. Dies ist auch durchaus

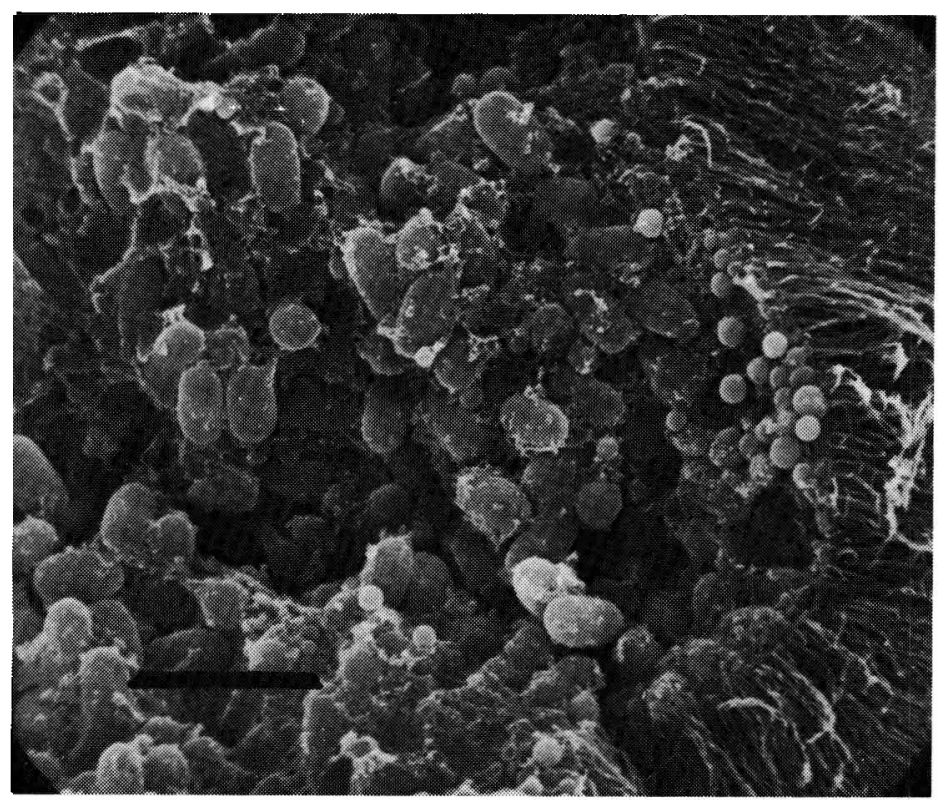

Авв. 13. - Mit Sporen infizierte Darmepithelzelle, in deren Randzone kleine Kugelstadien liegen. $10 \mu \mathrm{m}$

FIG. 13. - Cellule de l'épithélium intestinal infestée de spores.

Sur la zone marginale de la cellule on voit des stades boule. $10 \mu \mathrm{m}$ 


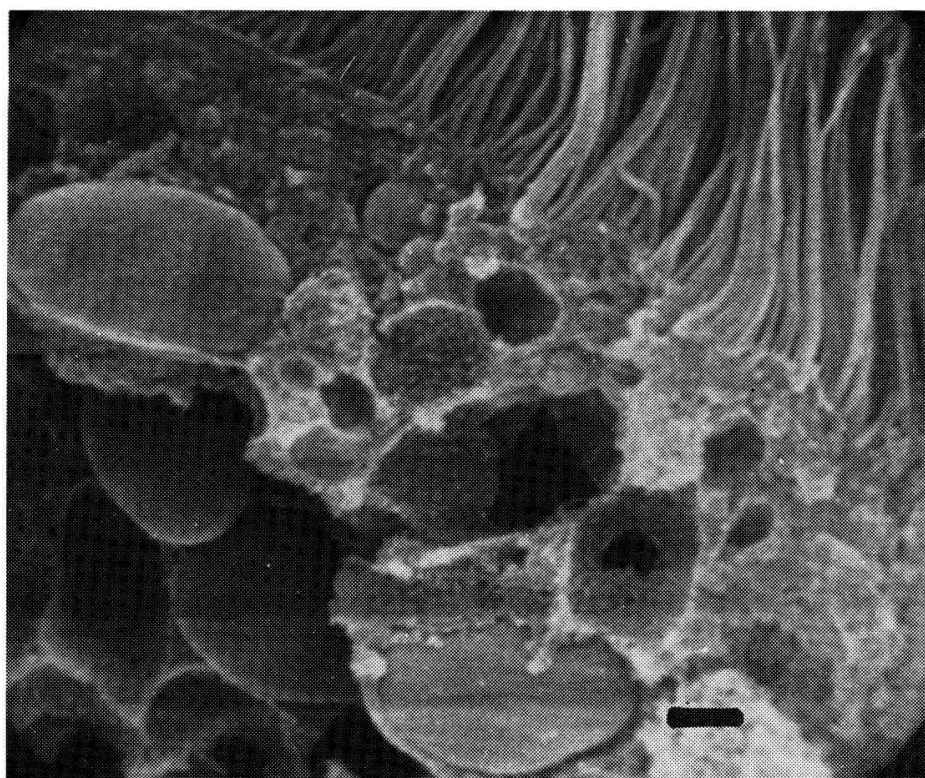

ABr. 14. - Siehe Abb. 13.

$1 \mu \mathrm{m}$

$1 \mu \mathrm{m}$

Fig. 14. - Voir Fig. 13.

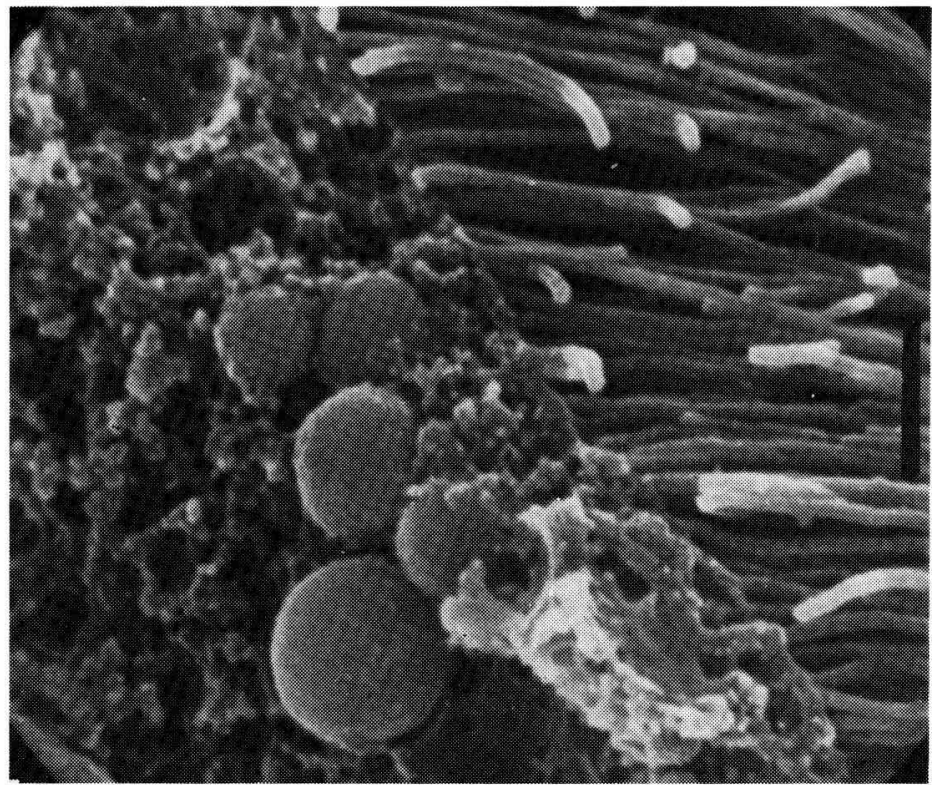

Aвв. 15. - Siehe Abb. 13.

$1 \mu \mathrm{m}$

Fig. 15. - Voir Fig. 13. 


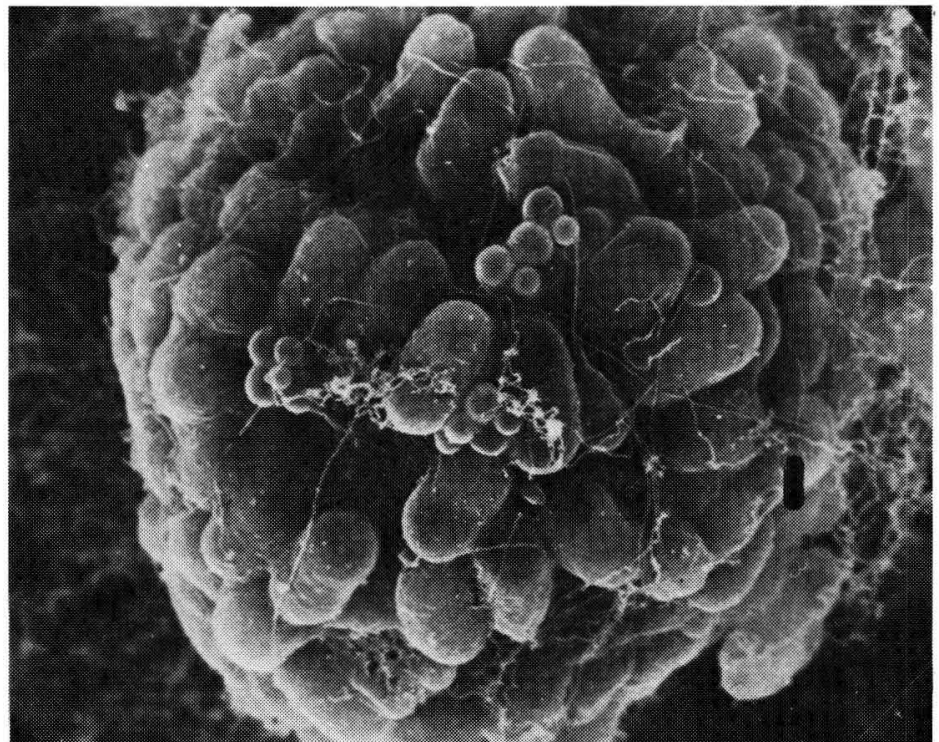

Авв. 16. - Mit Sporen infizierte, abgestossene Darmepithelzelle mit Kugelstadien im peripheren Bereich. $1 \mu \mathrm{m}$

FIG. 16. - Cellule de l'épithélium intestinal infestée de spores avec des stades boule dans la zone péripherique.

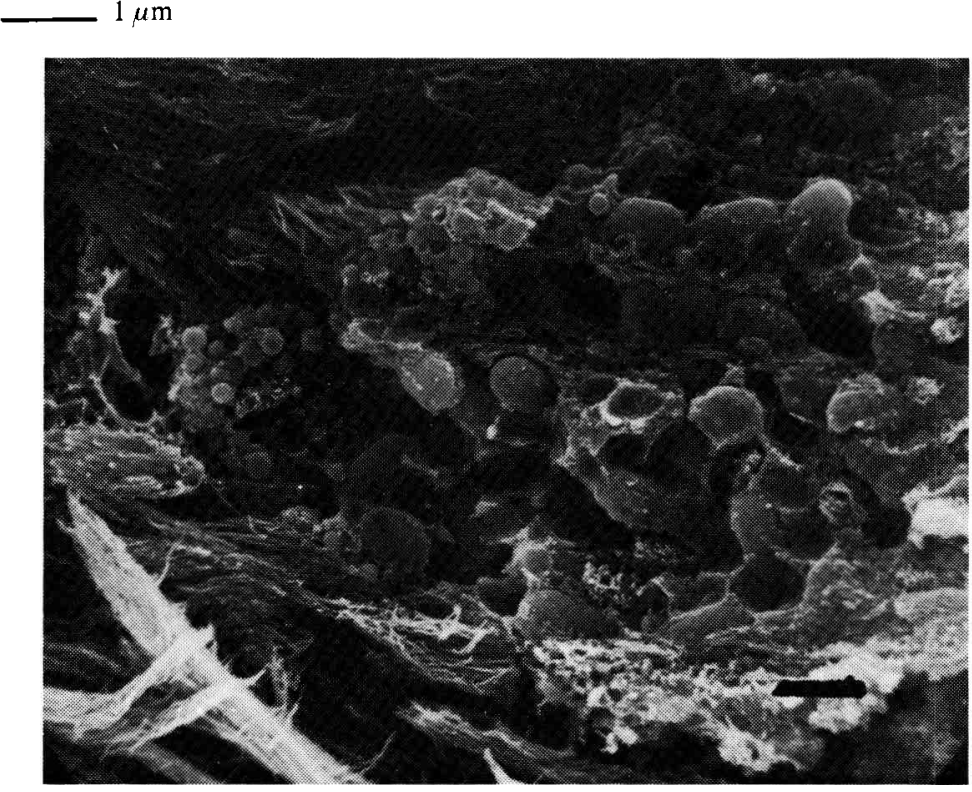

Авв. 17. - Sporeninfizierte Darmepithelzelle mit einem " Nest " von kleinen Kugelstadien.

$1 \mu \mathrm{m}$

Fig. 17. - Cellule de l'épithélium intestinal infestée de spores avec un " nid" de petits stades boule. 


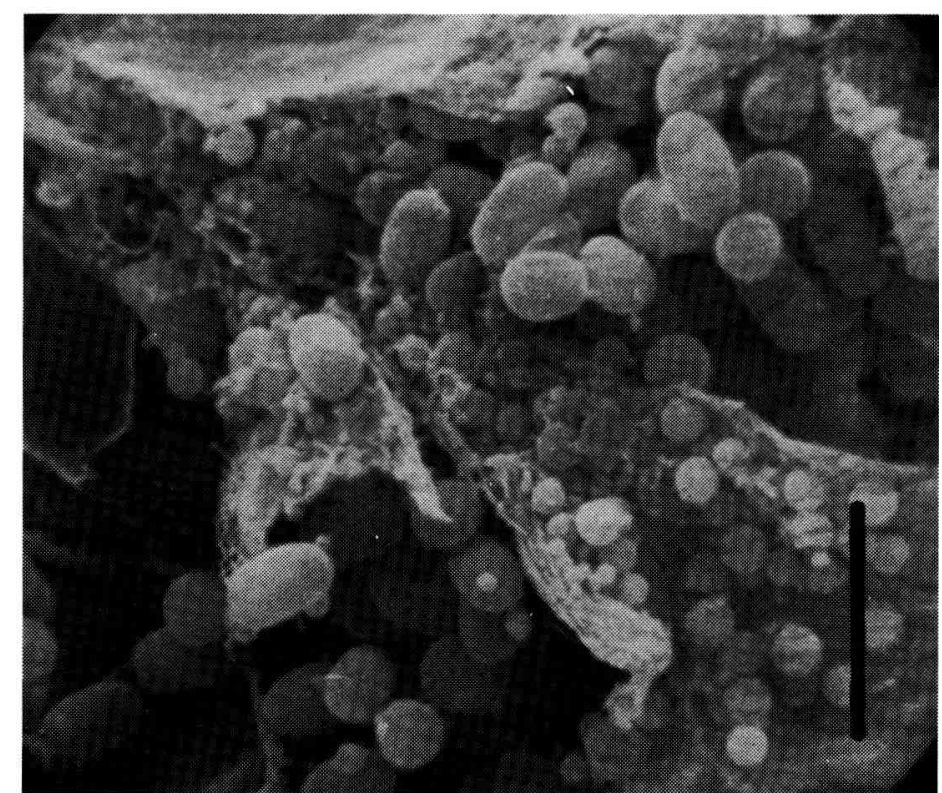

Aвв. 18. - Infizierte Darmepithelzelle mit Kugelstadien von unterschiedlicher Grösse. $10 \mu \mathrm{m}$

FIG. 18. - Cellule infestée de l'épithélium intestinal avec des stades boule de diverses tailles. $10 \mu \mathrm{m}$

naheliegend, da in dieser Aufbauphase mit Sicherheit ein intensiver Stoffaustausch zwischen dem Parasit und dem Zellplasma bestehen muss, durch den sich der Erreger die von ihm benötigten Aufbaustoffe aus dem Plasmakörper herausfiltert.

Diese Stadien der Aufbauphase, in deren Bereich wohl die bisher als Meronten, Sporonten, und Sporoblasten bezeichneten Stadien einzuordnen sind, variieren stark in Form und Grösse. Sie können relativ klein und noch fast kugelförmig sein (Meronten?), (siehe Abbildung 19 bis 21) aber auch grösser und vor allem breiter als die adulte Spore (siehe Abbildung 22 und 23). Wie stark Grösse und Form der Stadien variieren zeigt Abbildung 24, auf der auch zu erkennen ist, dass diese zumeist seitlich leicht eingebuchtet sind.

Neben solchen unterschiedlich grossen Entwicklungsstadien sind hin und wieder abnorm grosse Doppel- oder Riesensporen zu finden (Abbildung 25), die ja bereits von TRAPPMANN (1923) beschrieben worden sind.

Betrachtet man die Stadien des Erregers in den Darmepithelzellen, die gerade aus dem Zellverband abgestossen werden (siene Abbildung 26) oder bereits im Darmlumen liegen, so fällt einerseits auf, dass diese Stadien oftmals noch nicht bis zur adulten Spore ausgereift sind. Zum anderen findet man häufig zwischen den Sporenstadien kleine Kugelstadien. 


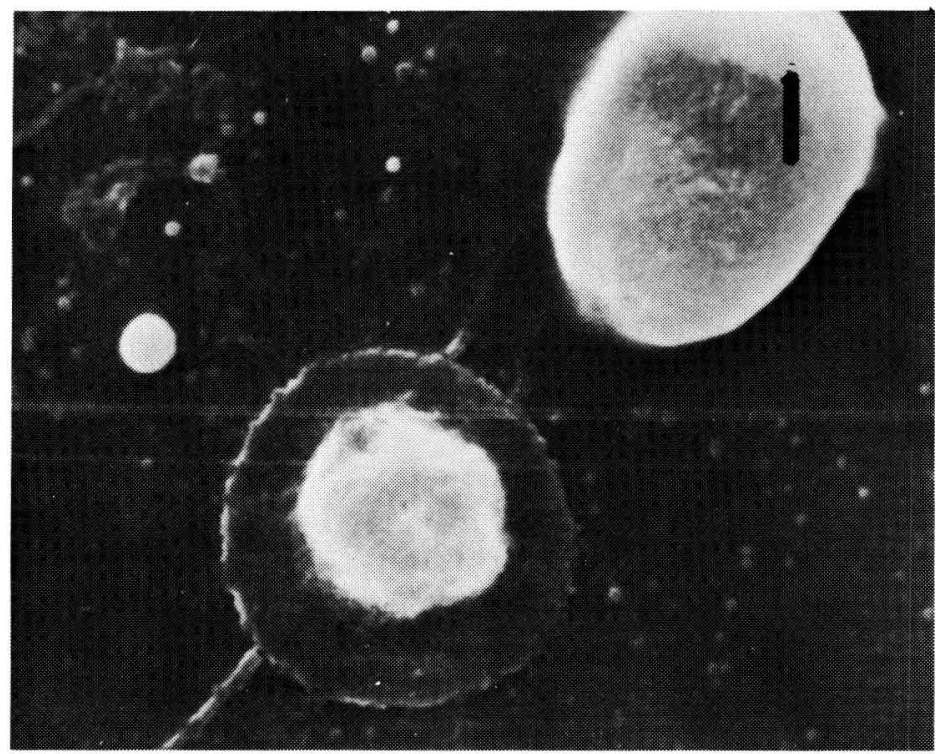

Aвв. 19. - Rundstadium im Ausstrich. Die Zellwand ist beim Antrocknen geschrumpft. $1 \mu \mathrm{m}$

FIG. 19. - Stade rond en frottis. La paroi cellulaire est ridée à cause du dessèchement. $1 \mu \mathrm{m}$

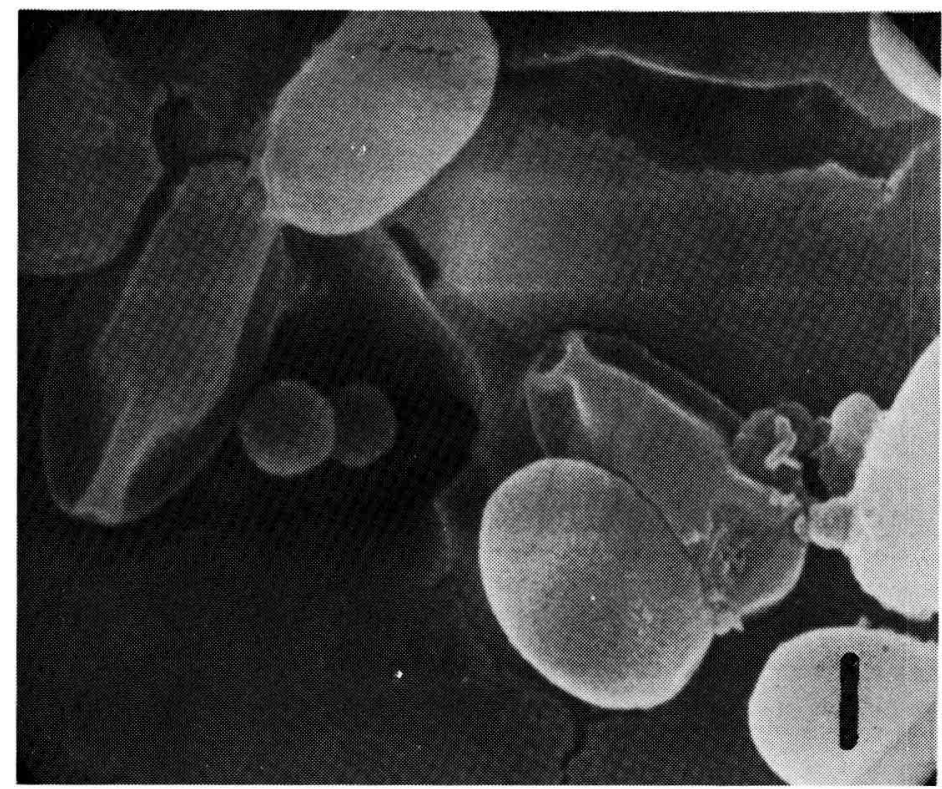

AвB. 20. - Angetrocknete und eingeschrumpfte Sporenbildungsstadien (zum Vergleich daneben adulte Spore)

$1 \mu \mathrm{m}$

FIG. 20. - Stades de formation de spores desséchées et ridées (comparer à côté la spore adulte). 


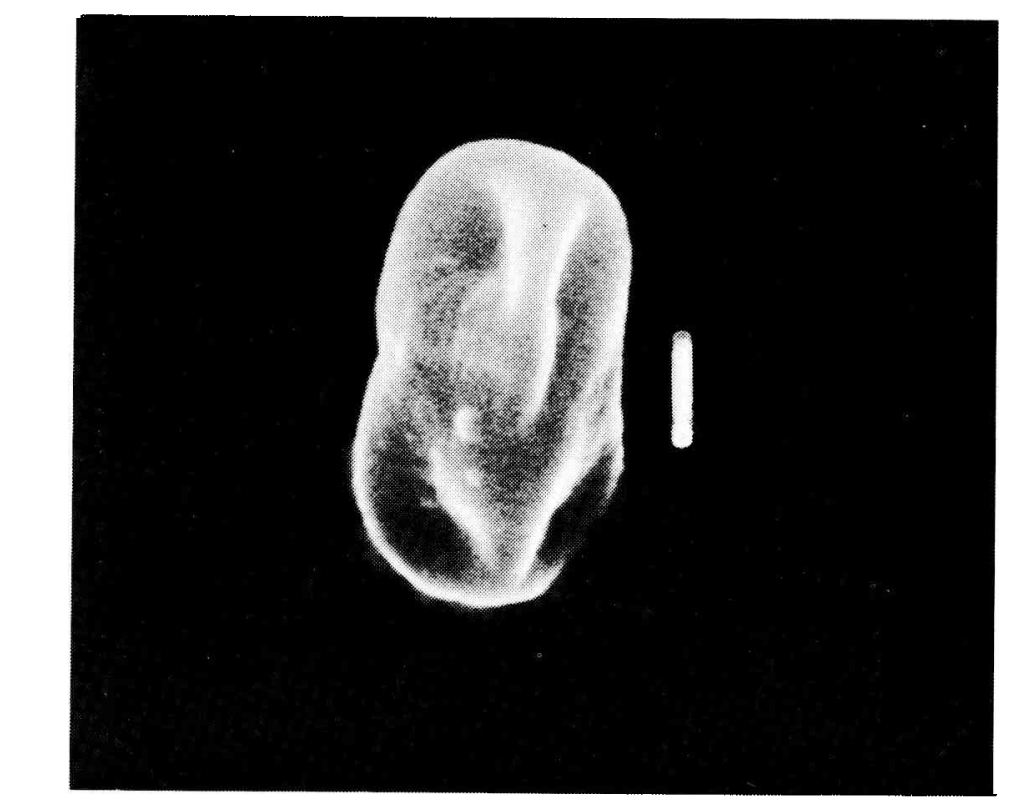

Авв. 21. - Siehe Abb. 20.

$1 \mu \mathrm{m}$

FIG. 21. - Vpir Fig. 20.

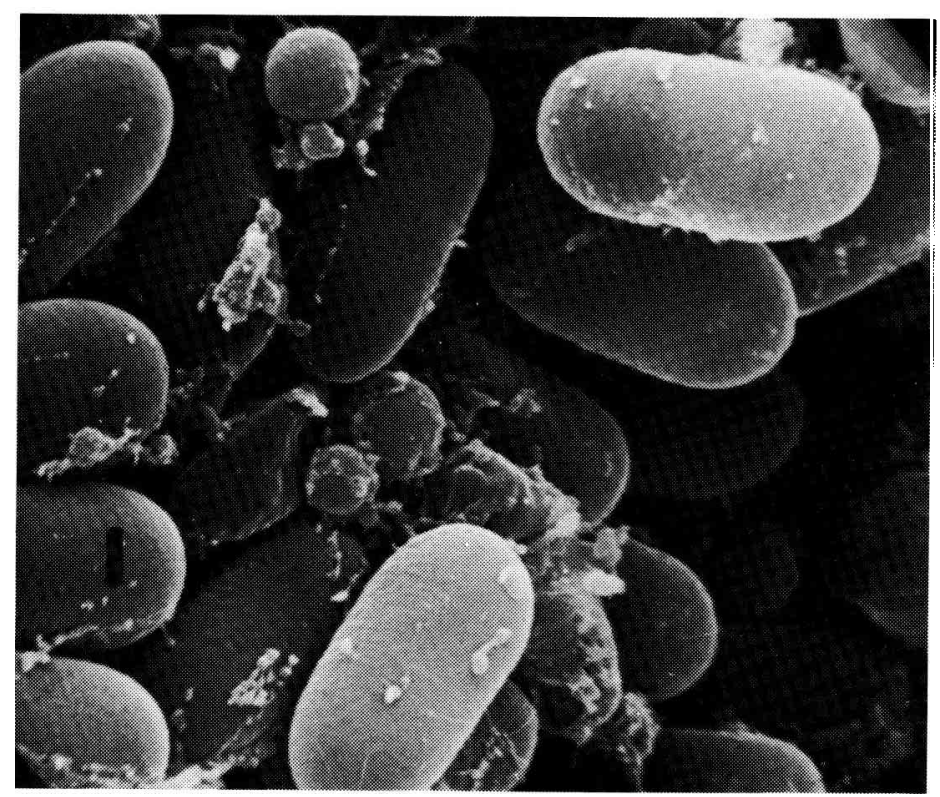

AвB. 22. - Stadien vor Abschluss der Sporenbildung.

$1 \mu \mathrm{m}$

FIG. 22. - Stades juste avant la formation des spores. 


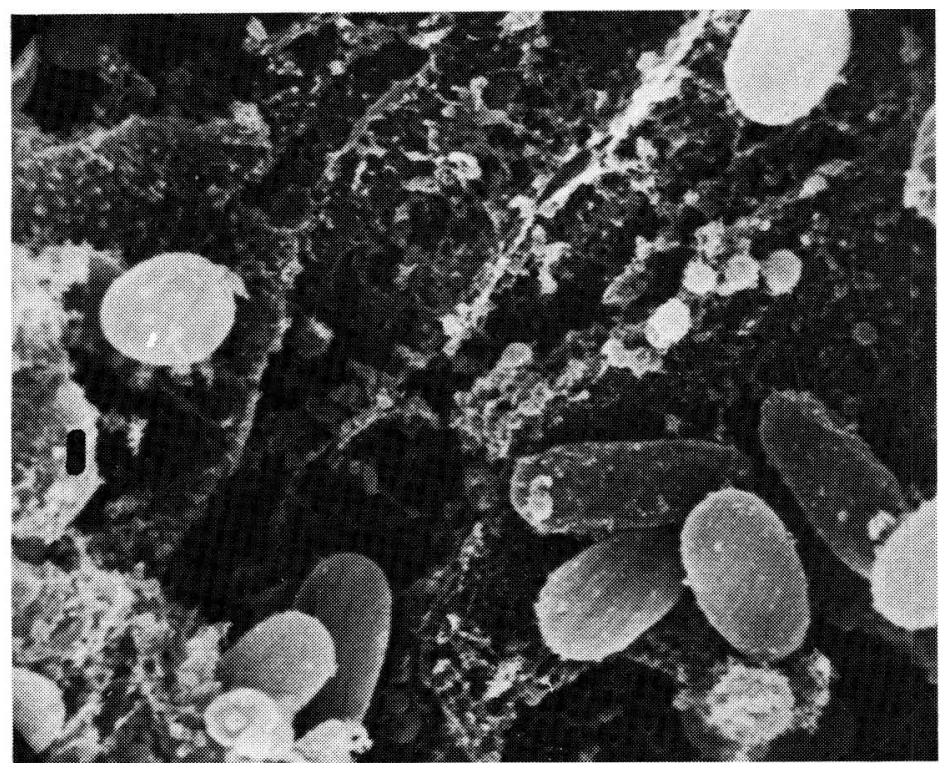

Авв. 23. - Siehe Abb. 22.

$1 \mu \mathrm{m}$

FIG. 23. - Voir Fig. 22.

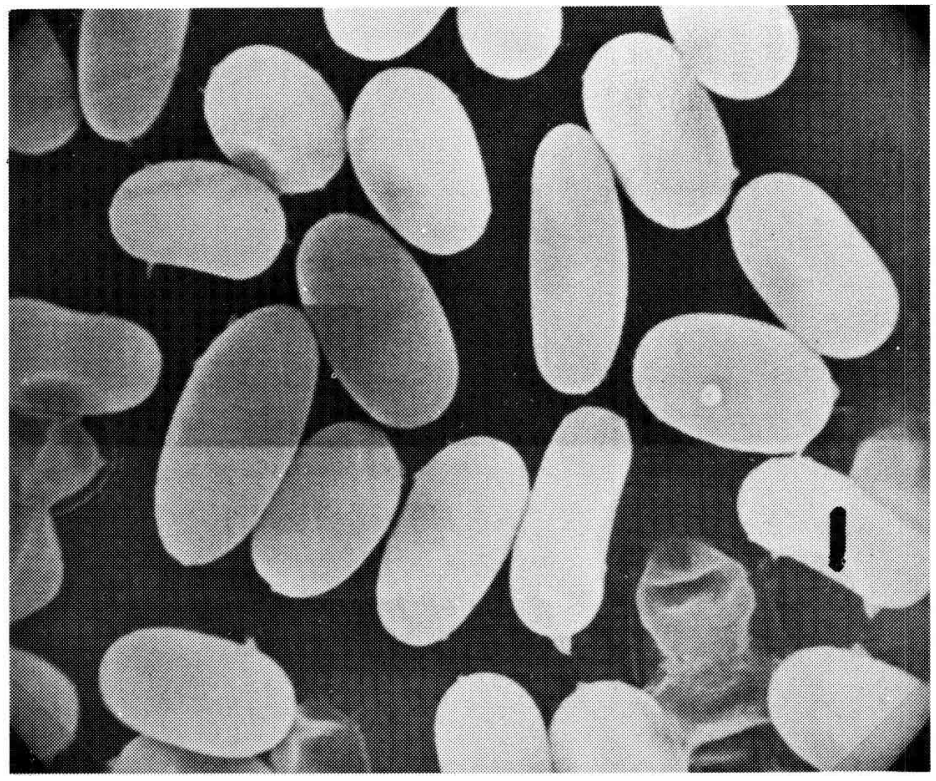

Aвв. 24. - Ein Ausstrich, der erkennen lässt, wie stark die Grösse der bereits sporenförmigen Stadien variiert, und der zeigt, dass diese Stadien teilweise seitlich eingebuchtet sind.

Fig. 24. - Frottis sur lequel on peut voir combien la taille des stades déjà sporulants varie et qui montre que ces stades sont en partie indentés sur le côté. 


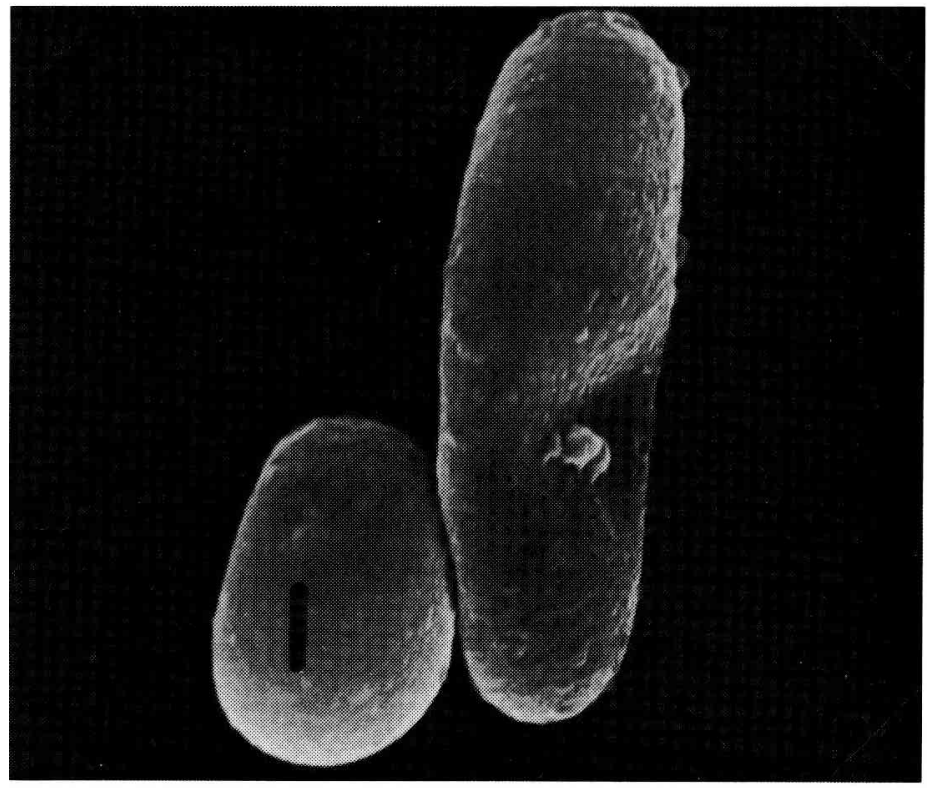

ABB. 25. - Riesenspore (nach TrappmanN).

$1 \mu \mathrm{m}$

FIG. 25. - Spore géante (d'après TRAPPMANN).

$1 \mu \mathrm{m}$

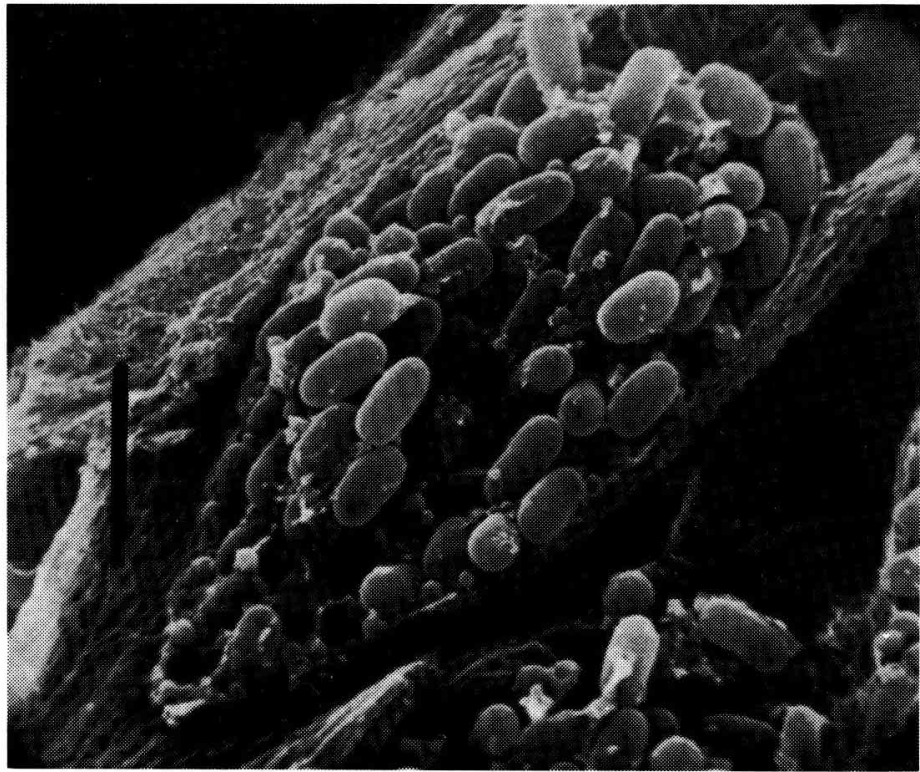

Aвв. 26. - Abgestossene Darmepithelzelle, die adulte Sporen und kleinste Rundstadien enthält. $10 \mu \mathrm{m}$

FIG. 26. - Cellule infectée de l'épithélium intestinal renfermant des spores adultes $10 \mu \mathrm{m}$ et les plus petits stades ronds. 
Die Reifung der Parasitenstadien fällt demnach nicht mit der Freisetzung in das Darminnere zusammen. Möglicherweise ist dadurch auf einfache Weise das Ausschleudern dieser zur Neuinfektion anderer Bienen gebildeter Stadien im Darm der Wirtsbiene verhindert.

Die Weiterverbreitung des Parasiten im Zellepithel der bereits infizierten Wirtsbiene, die Autoinfektion, über die LotMAR (1939) berichtet hat, könnte andererseits durch die am Anfang der Entwicklung stehenden kleinen runden Stadien (Planontenstadien) erfolgen, die bei dem Zerfall der ausgestossenen Zelle noch im Darm der Wirtsbiene wieder freigesetzt werden und in andere Zellen eindringen könnten.

\section{DISKUSSION}

Betrachtet man die durch die rasterelektronenmikroskopische Analyse gewonnenen Ergebnisse, so werden zumindest einige der bisher schwer zu deutenden Phänomene verständlich.

So war es kaum $\mathrm{zu}$ erklären, warum bei beginnender Infektion zumeist mehrere benachbarte oder dicht beieinanderliegende Zellen im hinteren Darmabschnitt zugleich infiziert wurden, worauf BoRCHERT (1924), GöTZE (1959), LOTMAR (1940) und STECHE (1960) bereits hingewiesen haben. Wenn, wie bisher angenommen, das Filament selbst bis in die einzelne Darmzelle eindringt, ist schwer erklärbar, wieso es dann zu einem gleichzeitigen Infektionsbeginn in mehreren benachbarten Zellen kommt.

Geht man nun davon aus, dass die Primärkeime zum Teil schon im Darmlumen freigesetzt werden und aktiv in das Zellepithel eindringen, so wird die Entstehung der beobachteten Infektionsinseln oder -Nester im Zellverband ohne weiteres verständlich. Eine einzige Spore, deren Filament einen Weg durch die peritrophischen Hüllen bis in die Nähe des Zellepithels hindurch gefunden hat, kann somit eine grössere Anzahl benachbarter Zellen gleichzeitig infizieren.

Dass austretende Filamente bei ihrem Heraustasten Hindernissen auszuweichen vermögen, oder besser gesagt beim Auftreffen auf ein Hindernis die Richtung ihres Vordringens ändern, hat STECHE (1960) nach Beobachtungen im Phasenkontratstmikroskop bereits beschrieben.

Des weiteren dürfte durch diese Untersuchungen ein Problem eine Klärung gefunden haben, welches sehr wahrscheinlich häufig zu Fehlschlüssen geführt hat. Wohl sicher haben die meisten mit der Nosema experimentierenden Kollegen kugelförmige Stadien, sei es beim histologischen Präparat in der Zelle eingebettet, sei es im Ausstrich einzeln oder in kleinerem Verband beobachtet.

Derartige Stadien wurden aufgrund der Aussagen von KöHLer (1921) zumeist als Kalkkörper angesprochen. So schreibt z.B. ZANDER (1946) folgendes : " Mit der 
Zerstörung des Zellkörpers geht ein Wechsel in der Menge und Zusammensetzung der verschiedenen Zelleinschlüsse einher. Die grossen lichtbrechenden, von Köhler als Kalkgebilde beschriebenen Körperchen nehmen erheblich ab oder verschwinden in seltenen Fällen ganz, während kleine schwach lichtbrechende, zuweilen unregelmässig gestaltete Körnchen sehr an Masse zunehmen, was HeRTIG (zit. nach ZANDER (1946) auf die Vermutung brachte, es könnte sich vielleicht um Lebewesen (Symbionten) handeln ». Unserer Meinung nach werden es wohl sicher die dargestellten Kugelstadien gewesen sein.

Im Zusammenhang mit den perlschnurartigen Ketten, die sich im ausgestülpten Filament nachweisen liessen (Abb. 4-7), ist eine Beobachtung von BORCHERT (1924) erwähnenswert, wobei sich allerdings die Frage stellt, wie er mit den damals zur Verfügung stehenden optischen Geräten derartige Vorgänge überhaupt ansprechen konnte. Leider hat er zur Methodik keine Angaben gemacht. Er berichtet folgendes : "Im vorderen Teile der jungen Spore sammelt sich das durch die Flüssigkeitsabgabe immer dichter werdende Zytoplasma mit den beiden Kernen an. Die Kerne lockern sich alsbald auf, so dass der vordere Sporenteil mit den Chromatinteilchen stark angefüllt ist. Von dieser Chromatinmasse treten Teilchen in die Vakuole ein und durchwandern die die Vakuole durchziehenden feinen Plasmastränge, wo sie oft als perlschnurartig nebeneinanderliegende Körnchen zu sehen sind. Zuweilen wandert diese " chromatische Substanz " in so starkem Masse in die Plasmastränge ein, dass sich die Körnchen zu einem dicken Knäuel zusammenballen. Das Plasma und die chromatische Substanz der die Vakuole durchziehenden Stränge und Schleifen wandeln sich in eine einheitliche, stark färbbare Substanz um, aus der sich schliesslich ein zusammenhängender Faden, der Polfaden bildet. Nachdem der grösste Teil der Chromatin-Masse in die Plasmastränge der grossen Vakuole abgewandert ist, bleiben im Amöboidkeim selbst zwei Kerne zurück ".

Nachzutragen bleibt zu diesem Zitat, dass BorCHERT noch von der Annahme ausging, der Amöboidkeim würde beim Austreten des Filamentes direkt die Spore verlassen, was - wie wir inzwischen mit Sicherheit sagen können - nicht der Fall ist.

Diese Beobachtungen von BORCHERT sind in Zusammenhang zu sehen mit Untersuchungen von G. SCHUBerT (1961). Er kommt auf Grund seiner elektronenmikroskopischen Befunde an Heterosporis finki (SCHUBERT) zu der Erkenntnis, dass im Entwicklungsprozess dieser Sporen Kerne auftreten, die sich dann jedoch auflösen, wobei Kernmaterial in das Plasma übergeht. Er beschreibt im hinteren Teil der Sporen Organelle, " die für die Infektion eines neuen Wirtes verantwortlich sein müssen (Abb. 27) ». Dementsprechend nennt er diesen Bereich den " generativen Teil ». In diesem befinden sich perlschnurartig aneinandergereihte Vesikel und Spiralstrukturen, ca. $12 \mathrm{~nm}$ dicke Fäden mit einem Windungsdurchmesser von etwa $45 \mathrm{~nm}$ aufgerollt. Abschliessend formuliert er folgendes Postulat: " $\mathrm{Da}$ irgendwie D.N.S. als Träger der Erbinformation vorhanden sein muss, liegt die Vermutung nahe, es könne sich bei den Spiralstrukturen um D.N.S. handeln. Die Tatsache, dass $20 \mathrm{~A}$ 


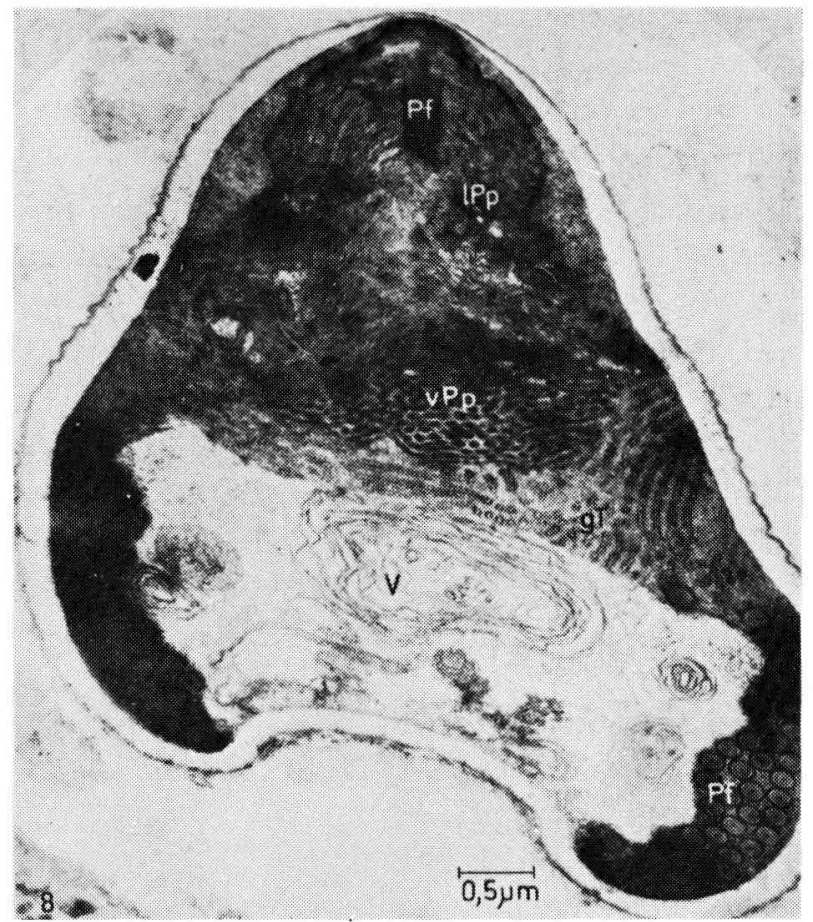

AвB. 27. - Unreife Spore von Heterosporis finki SchuBERT.

gT : generativer Teil; LPp : lamellärer Teil des Polaroplasten; Pf : Polfaden;

V: Vakuole; vPp : vesikulärer Teil des Polaroplasten (nach SchuBERT).

FIG. 27. - Spore immature d'Heterosporis finki SchUBERT.

$\mathrm{gT}$ : partie reproductrice; $\mathrm{LPp}$ : partie lamellaire du polaroplaste; Pf : filament polaire;

$\mathrm{V}$ : vacuole; vPp : partie vésiculaire du polaroplaste (d'après SCHUBERT).

Fäden als Untereinheiten aufgelöst wurden, unterstützt die Hypothese. Die Gebilde erinnern bis zu einem gewissen Grad an die in Amöben-Kernen gefundenen, die sicher D.N.S.-Helices darstellen (BRANDT u. PAPPas, 1961) ».

Möglicherweise, oder man darf wohl sagen, wahrscheinlich handelt es sich bei der in den Polfäden nachgewiesenen Substanz um diese von SCHUBerT aufgezeigten Bestandteile der Sporen.

Die vorliegenden Untersuchungen, bei denen Abertausende infizierter Zellen im Rasterelektronenmikroskop betrachtet wurden, gestatten es des weiteren, auf eine Tatsache hinzuweisen, die sich unter Anwendung histologischer Verfahren nur schwer beurteilen liess. Gemeint ist die Frage, ob infizierte Zellen generell restlos mit Sporen angefüllt sind, oder ob es auch Zellen mit einem geringeren Füllungsgrad gibt.

Bisher ging man ja von der These aus, dass sich die schon weiter fortgeschrittenen Stadien solange zu teilen vermögen, bis der Vorrat an Zellplasma verbraucht ist. Man nahm also an, dass jede befallene Zelle bis zum Bersten mit Sporen ausgefüllt ist. 
Es hat sich nun aber gezeigt, dass es sehr wohl infizierte Zellen gibt, noch im Zellverband oder auch schon abgestossen im Darmlumen, die keineswegs restlos mit Sporen angefüllt sind. Die mehr oder weniger rein mechanistische Vorstellung der Steuerung dieses Problems dürfte somit wohl $\mathrm{zu}$ einfach sein.

Abschliessend sei die Frage angesprochen, ob der Terminus " Nosema » überhaupt zutreffend ist. Besonders Morgenthaler hat verschiedentlich auf diese Problematik hingewiesen.

Wenn wir der Gliederung von J. WeISER (1961) folgen, dann haben " die Gattungen Pérezia und Glugea beide als Merkmal, dass im Gegensatz zu Nosema, ihre Sporonten zwei Sporoblasten und schliesslich auch zwei Sporen bilden, wobei Glugea ausschliesslich Parasiten der Vertebraten, Pérezia dagegen Parasiten der Evertebraten umfasst ". Da die vorliegenden Untersuchungen nun mit Sicherheit ausschliessen, dass es speziell vor der Bildung der Spore zu Teilungsvorgängen der ganzen Stadien kommt, lässt sich jetzt zweifelsfrei feststellen, dass nach dem System der Mikrosporidia von WEISER (1961) der Parasit folgendermassen einzuordnen und zu benennen ist :

Ordung : Microsporidia, BALBIANI, 1882.

Unterordnung : Monocnidea, LÉGER u. HESSE, 1922.

Familie : Nosematidae, LABBÉ, 1899.

Gattung : Nosema, NÄGELI, 1957.

Art : Nosema apis, ZaNDER, 1909.

Eingegangen im Februar 1981.

Reçu pour publication en février 1981.

\author{
RÉSUMÉ \\ ÉTUDE AU MICROSCOPE ÉLECTRONIQUE A BALAYAGE DE L'ONTOGENÈSE \\ DE NOSEMA APIS ZANDER
}

Afin de savoir à quelles phases du développement de Nosema apis Zander ont lieu les divisions des stades parasites, on a étudié en microscopie électronique à balayage l'agent pathogène à différentes périodes de développement dans l'épithélium intestinal d'abeilles contaminées et sur des préparations de frottis.

Dans ce but, on a déshydraté des intestins d'abeilles, des frottis d'épithélium intestinal d'abeilles infectées et des spores pré-traitées au Ringer entomologique selon les procédés habituels à l'acétone; on les a desséchés au point critique et finalement métallisés à l'or. Avant d'être posés sur le porte-objet les intestins ont été ouverts en divers endroits de façon à pouvoir analyser leur épithélium et leur contenu. Contrairement à ce que l'on attendait d'après les données bibliographiques existantes, les stades observables en lumière optique n'ont montré aucune division. Ce n'est qu'au début du développement, juste après la sortie hors du filament polaire des stades infectieux fragmentés en très fins segments, que se produit une phase de division, très certainement souvent encore dans la lumière intestinale. Les stades libres migrent alors dans l'épithélium intestinal. Ceci permet de comprendre l'infection simultanée de plusieurs cellules épithéliales voisines, c'est-à-dire la formation de ce qu'on appelle nids d'infection.

On distingue simplement à la structure extérieure les stades primaires difficilement observables même en microscopie électronique, ainsi que les stades de diverses tailles qui forment des boules et des spores. 
A côté des cellules épithéliales généralement remplies de spores se trouvent çà et là des cellules intestinales faiblement remplies de ces stades.

L'hypothèse, selon laquelle la division des stades parasites est uniquement dirigée par la fourniture en plasma cellulaire, est donc assurément fausse.

Puisqu'aucune division cellulaire n'a lieu avant la formation de spores adultes, la classification systématique du parasite en Nosema apis Zander est exacte.

\section{LITERATUR VERZEICHNIS}

BORCHERT A., 1924. - Die seuchenhaften Krankheiten der Honigbiene. Verlagsbuchhandlung Richard Schoetz/Berlin.

BRandt P. W. and Pappas G. D., 1961. -- The fine structure of helices in Amoeba nuclei. Progress in Protozoology. Proc. J. Int. Conf. Protozool, 408-509, Prague 1961.

Fantham H. B. and Porter A., 1912. - The morphology and Life history of Nosema apis and the significance of its various stages in the so-called Isle of Wight disease in bees (Microsporidioses), Ann. Trop. Med. Paras., (6) : 163-195.

Goetze G., Eberhardt F., Zeutzschel B., 1959. - Versuche zur Selbstheilung und Therapie der Nosematose der Honigbiene. Bonn, Inst. für Bienenkunde der Universität.

JACOBS F., 1976. - Lichtmikroskopische Untersuchungen der Nosema apis (Mikrosporidia) und deren morphologische Aspekte. Biologische Aspekte der Nosema (Symposium Merelbeke) ApimondiaVerlag, Bukarest.

KöHLER A., 1921. - Über die Einschlüsse der Epithelzellen des Bienendarms und die damit in Beziehung stehenden Probleme der Verdauung. Zeitschr. f. angew. Entomologie, Bd. VII, (1): 68-91.

LotMAR R., 1939. - Untersuchungen über die Zahl der Sporen in nosemakranken Bienen. Schweizer. Bienenzeitung, (9) : 39.

Lotmar R., 1940. - Beiträge zur Pathologie des Bienendarmes. Landw. Jahrb. d. Schweiz, 1940, 803.

Peters W., 1968. - Elektronenmikroskopische Untersuchungen an chitinhaltigen Strukturen. Zoologischer Anzeiger, (31): 681-695.

SCHUBERT G., 1969. - Ultracytologische Untersuchungen an der Spore der Mikrosporidienart, Heterosporis finki, gen. n. sp. n. Zeitschr. Parasitenkunde, (32): 59-79.

SCHUBERT G., 1969. - Elektronenmikroskopische Untersuchungen zur Sporonten-Sporenentwicklung der Mikrosporidienart Heterosporis finki. Zeitschr. Parasitenkunde, (32) : 80-92.

SteChe W., 1960. - Ätiologie und Therapie der Nosematose der Honigbiene. Zeitschr. f. Bienenforschung, $5(3): 49-92$.

STEChe W., 1962. - La Nosemose des Abeilles. Bulletin Apicole, Tome IV, (1) : 67-83.

SteChe W., Held Th., 1978. - Rasterelektronenmikroskopische Aufnahmen des Parasiten. Nosema apis Zander. Allg. dtsch. Imkerztg., (1) : 323-328.

TrappmanN W., 1923. - Die Nosemaseuche der Honigbiene unter besonderer Berücksichtigung des Erregers. Zentralblatt $f$. Bakteriologie, Parasitenkunde und Infektionskrankheiten II, Bd., (68) 27-49.

WeISer J., 1961. - Die Mikrosporidien als Parasiten der Insekten. Paul Parey, Hamburg/Berlin.

ZANDER E., 1909. - Tierische Parasiten als Krankheitserreger bei der Biene (Vortrag in Weissenfels am 9.8.1909). Leipziger Bienenzeitung, 24 (10) : 147-150 und (11) : 164-166.

ZANDER E., 1946. - Krankheiten und Schädlinge der erwachsenen Biene. 5. Aufl., E. Ulmer, Stuttgart. 